\title{
Non-ohmic Mott conductivity and thermometric characteristics of heavily doped silicon structures
}

\author{
Yu.M. Shwarts \\ Institute of Semiconductor Physics, NAS Ukraine, 45 Prospect Nauki, Kyiv, 03028, Ukraine \\ Tel./Fax: (380-44) 265-56-70; E-mail: shwarts@isp.kiev.ua
}

A.V. Kondrachuk, M.M. Shwarts, L.I. Shpinar

Institute of Physics, NAS Ukraine, 46 Prospect Nauki, Kyiv, 03028, Ukraine

Tel.: (380-44) 265-72-71, (380-44) 265-08-23

\begin{abstract}
We investigated $I-V$ curves and thermometric characteristics of heavily doped $p$ silicon structures at liquid helium temperatures. The variable-range hopping conductivity is shown to occur in the structures studied. The obtained parameters of non-ohmic Mott conductivity and their temperature and electric field dependencies enabled us to explain the features in structure thermometric characteristics. It is shown that both height and position of the responsivity peak depend on the extent to which the hopping conductivity is non-ohmic. They are essentially determined by the density of states near the Fermi level.
\end{abstract}

Keywords: non-ohmic Mott conductivity, heavily doped silicon structures, liquid helium temperatures, thermometric characteristics, responsivity.

Paper received 24.02.00; revised manuscript received 04.06.00; accepted for publication 16.06.00.

\section{Introduction}

The silicon diode sensors are widely used in modern cryogenic thermometry $[1,2]$. Their main characteristics are (i) thermometric characteristic, i.e., temperature dependence, $U(T)$, of the voltage drop $U$ on a diode through which a continuous direct current $I$ flows and (ii) responsivity, $d U / d T$. At low temperatures a non-monotonic responsivity change with temperature and its abrupt (tens-hundreds of $\mathrm{mV} / \mathrm{K}$ ) jump are observed. Such a behavior of thermometric characteristic in the low-temperature region is typical also of the Ge-, GaAs- and GaAlAs-based diode temperature sensors. It is related to a change in the current flow character in diode structures, or, in other words, to an active role of the series resistance of diode sensor base.

Up to now, the mechanisms for current flow that determine formation of thermometric characteristics at low temperatures are the least understood. At liquid helium temperatures conduction occurs through hopping over impurity states. So it is particularly sensitive to the features in the density of states energy distribution over semi- conductor gap [3, 4]. The compensation degree and doping levels in a semiconductor determine the spread in both spacing between the electron localization centers and binding energies of electrons on the impurity centers. This permits occurrence of different (activation, as well as variable-range) hopping conductivity mechanisms [5].

When explaining experimental data concerning temperature dependence of conductivity $\sigma$ in heavily doped silicon, the authors of [5] used the concept of a hopping conductivity mechanism whose temperature dependence is $\sigma \sim \exp \left(T^{-1 / 2}\right)$. Such dependence is related to the presence of a Coulomb gap at the Fermi level. At the same time the results of simulation show [3] that at liquid helium temperatures the Coulomb gap is spreading, and this should result in the $\sigma \sim \exp \left(T^{-1 / 4}\right)$ dependence.

Our work deals with investigation of low-temperature current flow mechanisms in test structures that serve as a basis for silicon diode temperature sensors, as well as determination of physical parameters that are responsible for thermometric characteristic formation and structure responsivity at liquid helium temperatures. 


\section{Samples and experimental technique}

We investigated test $p$-silicon resistors. They were prepared using boron diffusion to a semi-insulating substrate. The boron surface concentration was about $\sim 3 \cdot 10^{18} \mathrm{~cm}^{-3}$. The resistor length was from 17.5 to $52.5 \mu \mathrm{m}$.

The experimental studies of resistor thermometric characteristics were performed at the automated certified bench UGT-A. The operating current was maintained at a required level with accuracy better than $\pm 0.05 \%$. The maximum value of main absolute error when measuring temperature was $\pm 0.03 \mathrm{~K}$ over the 4.2 to $373 \mathrm{~K}$ temperature range.

The resistor $I-V$ curves were taken at the automated bench over the 4.2 to $13 \mathrm{~K}$ temperature range and $10^{-11}$ to $10^{-2}$ A current range. The thermostatic control of the samples studied was performed in liquid helium vapors. In this case the temperature level was maintained with accuracy better than $\pm 0.01 \mathrm{~K}$. When measuring voltage (current), error was below $\pm 5 \mathrm{mV}( \pm 5 \%)$.

\section{A model for resistor low-temperature con- ductivity}

The boron impurity concentration $N_{\mathrm{B}}$ in the resistors studied was $3 \cdot 10^{18} \mathrm{~cm}^{-3}$ (with the compensation degree $K<<1$ ). So the spacing between impurity atoms $\left(N_{\mathrm{B}}\right)^{-1 / 3}>a$ where $a(23 \AA)$ is the radius of the state of a hole localized on a boron atom. It is known [6] that in doped semiconductors at low temperatures most of the impurity atoms are neutral, if $N a^{3}<<1$ and the compensation degree $K<<1$. Only small amount of them take part in the current flow process. In this case the charge carrier transitions between the atomic states involve phonons. When temperature goes down, then both the phonon number and energy decrease, and charge carriers hopping between states that are closer in energy (even if they are spaced wider) becomes more preferable than that between the nearest neighbors whose energies differ substantially. This mechanism is known as variable-range hopping conductivity, or Mott conductivity. Mott has shown [4] that such a conductivity is realized when hopping occurs between the states lying in a narrow energy range near the Fermi level. According to [6], a width of such an optimal range $\varepsilon_{0}$ is:

$\varepsilon_{0}(T)=(k T)^{3 / 4} /\left[g(\mu) a^{3}\right]^{1 / 4}$.

Here $g(\mu)$ is the density of states at the Fermi level $\mu$, $k$ is the Boltzmann constant and $T$ is temperature. In this case conductivity $\sigma$ is of the following form [4]:

$\sigma=\sigma_{0} \exp \left[-\left(\frac{T_{0}}{T}\right)^{1 / 4}\right]$

where

$T_{0}=b / a^{3} g(\mu) k$.
Here $b$ is a numerical coefficient that is determined in the percolation theory, and $s_{0}$, according to theoretical estimation [6], is:

$$
\begin{aligned}
& \sigma_{0}=\left(9 \pi a^{3} d s^{5} h^{4} \chi^{2} / 4 E_{1}^{2} e^{6}\right)^{-1}\left(T_{0} / T\right)^{(2-v) / 4}= \\
& =\sigma_{00}\left(T_{0} / T\right)^{(2-v) / 4}=\sigma_{0 T}
\end{aligned}
$$

Here $d$ is the crystal density; $e$ is the electron charge; $h$ is the Planck constant; $s$ is the sound velocity in a crystal; $\chi$ is permittivity; $E_{1}$ is the deformation potential constant; $v$ is an index that determines correlation radius for an infinite cluster in the percolation theory (in the threedimensional case it is 0.9 ).

When temperature goes down, then the mean hopping range $R$ grows as

$$
R=(a / 2)\left(T_{0} / T\right)^{1 / 4} \text {. }
$$

In low electric fields Mott conductivity is ohmic. The energy gained by a charge carrier at the hopping range $R$ grows with electric field and may become comparable to $k T$. The following two electric field ranges may be distinguished where ohmic type of conductivity breaks: $k$ T/e $a>E>k$ T/e $R$ (intermediate fields) and $E>>k$ Tlea (high fields) [7]. For intermediate electric fields an approximate expression for the non-ohmic Mott conductivity is $[8,9]$ :

$$
\sigma=\sigma_{0} \exp \left[-\left(\frac{T_{0}}{T}\right)^{1 / 4}+\alpha(T) E\right]
$$

where $\alpha(T)=R C e / k T$, and $C$ is a constant. Then

$$
\alpha(T)=\alpha_{0} T^{-5 / 4}=C e(a / 2)\left(T_{0}\right)^{1 / 4} k^{-1} T^{-5 / 4} .
$$

The expression (6) was obtained using the concept of an optimal range of density of states [4]. So this expression is very crude at intermediate and high electric fields, since in this case the symmetry of the problem studied is violated. However, the electric field dependence of conductivity given by (6) has been confirmed experimentally for various semiconductors $[10,11]$. The same field dependence has been obtained also using computer simulation of the corresponding problem in percolation theory [7]. In this case it was found that parameter $C$ depends on

temperature $T$ but slightly, $C=(14 \pm 2) \cdot 10^{-3}\left(\frac{T_{0}}{T}\right)^{1 / 4}$, and its values lie between 0.21 and 0.39 .

\section{A comparison between the model for resistor low-temperature conductivity and experiment}

An analysis of the test resistor conductivity in the 4.2 to $13 \mathrm{~K}$ temperature range was based on comparison between (i) the theoretical and experimental $\sigma(E)$ curves at 
a constant temperature value and (ii) theoretical and experimental $\sigma(T)$ curves at a constant electric field value. The experiment has proved that $\sigma(E, T)$ did not depended on the distance between contacts. Therefore, conductivity was determined by the properties of sample bulk.

Conductivity $\sigma$ vs electric field $E$ curves at different temperatures are shown in Fig. 1. From them one may conclude that conductivity is non-ohmic. It may be presented, to a high degree of accuracy, as

$\ln [\sigma(E, T)] \sim \alpha(T) E$

Let us determine the temperature dependence of parameter $\alpha$ in expression (8) using the results given by Fig. 1. One can see from Fig. 2 that the experimentally found $\alpha(T)$ curve coincides with the theoretical one and is of the form $\alpha(T)=\alpha_{0} T^{-5 / 4}$, where $\alpha_{0}=0.03$. Using expression (7), one can write down the $\sigma(E, T)$ function as

$\sigma(E, T)=\sigma_{0} \exp \left\{C e(a / 2)\left(T_{0} / T\right)^{1 / 4} \times\right.$

$\left.\times(k T)^{-1} E-\left(T_{0} / T\right)^{1 / 4}\right\}$

Shown in Fig. 3 are both experimental and theoretically calculated $\ln \sigma(T, E)$ curves for three values of electric field. One can see that at electric fields $E \sim 10^{2} \mathrm{~V} / \mathrm{cm}$ conductivity is rather accurately described by the Mott law (2) over the whole temperature range studied. From this one may estimate the parameter $\left(T_{0}\right)^{1 / 4} \approx 36^{1 / 4} \mathrm{~K}^{1 / 4}$.

With allowance made for expression (5), the condition for the ohmic character of Mott conductivity to break, $E>k T / e R$, is of the following form: $E>k T^{5 / 4} / e(a / 2) \times$

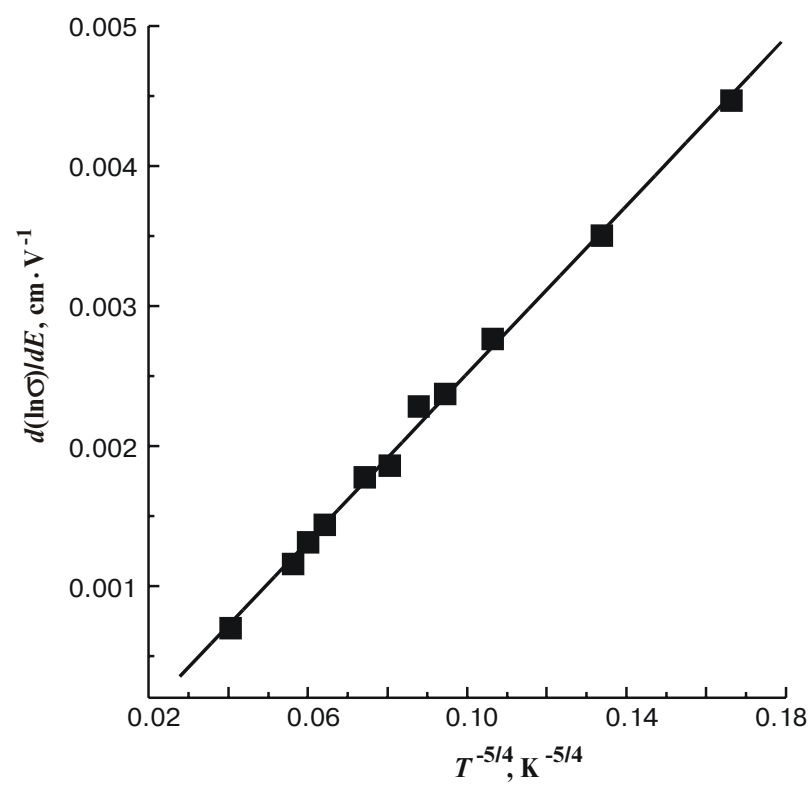

Fig. 2. Temperature dependence of the coefficient $\alpha$ : dots experiment, lines - theory.

$\times\left(T_{0}\right)^{1 / 4}$. The corresponding estimation gives $E>30 \times$ $\times(T)^{5 / 4} \mathrm{~V} / \mathrm{cm}$. Since it is supposed [7] that expression (8) holds for electric fields $E<k T / e a$, one gets: $30 \cdot(T)^{5 / 4} \mathrm{~V} / \mathrm{cm}$ $<E<500 \cdot T \mathrm{~V} / \mathrm{cm}$. These criteria are obeyed over the whole temperature range studied.

Using the experimentally found values of parameters $T_{0}$ и $\alpha_{0}$ and their analytical expressions (3) and (7), one



Fig. 1. Conductivity $\sigma$ vs electric field $E$ curves at different temperatures.

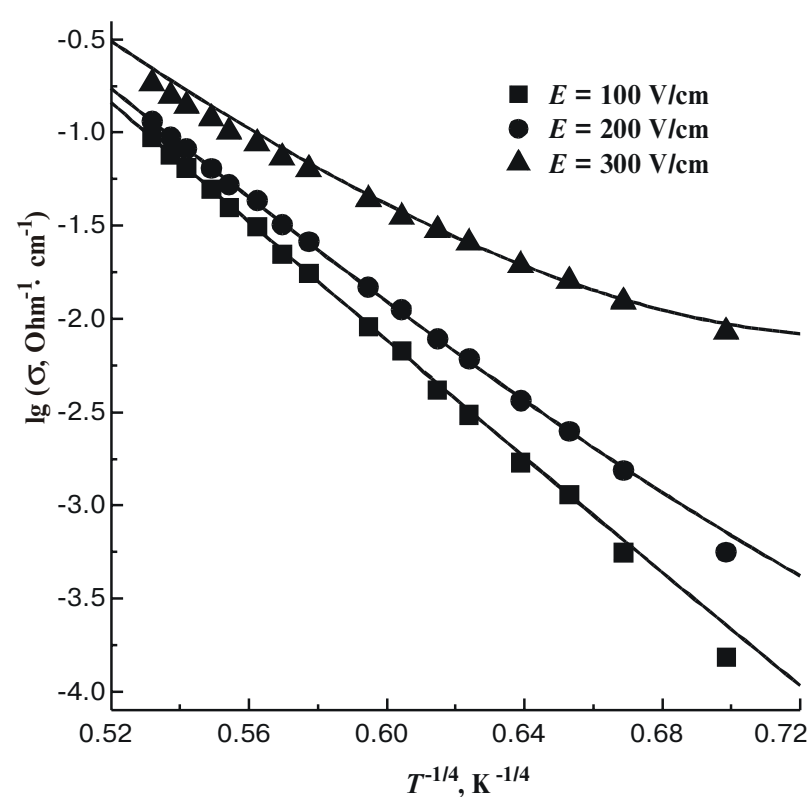

Fig. 3. Logarithm of conductivity vs $T^{1 / 4}$ curves at different electric fields: dots - experiment, lines - calculated with expression (9). 


\section{Yu. M. Shwarts et al.: Non-ohmic Mott conductivity and thermometric characteristics ...}

can determine $C$ in expression (7): $C=0.3$. This is in agreement with the results of [7]. One can also determine from expression (3) the density of states at the Fermi level: $g(\mu) \approx 9.6 \cdot 10^{18} \mathrm{eV}^{-1} \mathrm{~cm}^{-3}$.

Now, after all the parameters in expression (9) have been determined, one can obtain theoretical expressions for $\sigma(E, T)$ and compare them to the experimental results found at different electric field values. Fig. 3 illustrates the degree of agreement between the dependencies calculated from expression (9) and experimental data.

A comparison between the theoretical $\left(\sigma_{0 \mathrm{~T}}(4)\right)$ and experimental $\left(\sigma_{0 \mathrm{E}}\right)$ values of the pre-exponential factor in the expression for conductivity may serve as an additional check for Mott type of current flow in the test resistors studied. Our estimations (made for $T=10 \mathrm{~K}$ ) give: $\sigma_{0 \mathrm{~T}} \approx 9.7 \cdot 10^{6} \Omega^{-1} \mathrm{~cm}^{-1}, \sigma_{0 \mathrm{E}} \approx 10^{7} \Omega^{-1} \mathrm{~cm}^{-1}$.

Thus, a comparison between the results of experimental investigations of low-temperature conductivity for test resistors and theoretical description of non-ohmic Mott conductivity shows that they are in satisfactory agreement.

\section{Thermometric characteristics of test resistors}

From the model considered it follows that the $I$ - $V$ curve of the test resistor may be presented as

$$
I=\frac{S}{L} \sigma_{00}\left(\frac{T_{0}}{T}\right)^{\frac{2-v}{4}} U \exp \left(\alpha_{1} T^{-5 / 4} U\right) \exp \left[-\left(\frac{T_{0}}{T}\right)^{\frac{1}{4}}\right]
$$

Here $\alpha_{1}=\alpha_{0} / L ; L$ is the spacing between the test resistor contacts; $S$ is the resistor cross-section area; $U$ is the voltage applied to the sample studied.

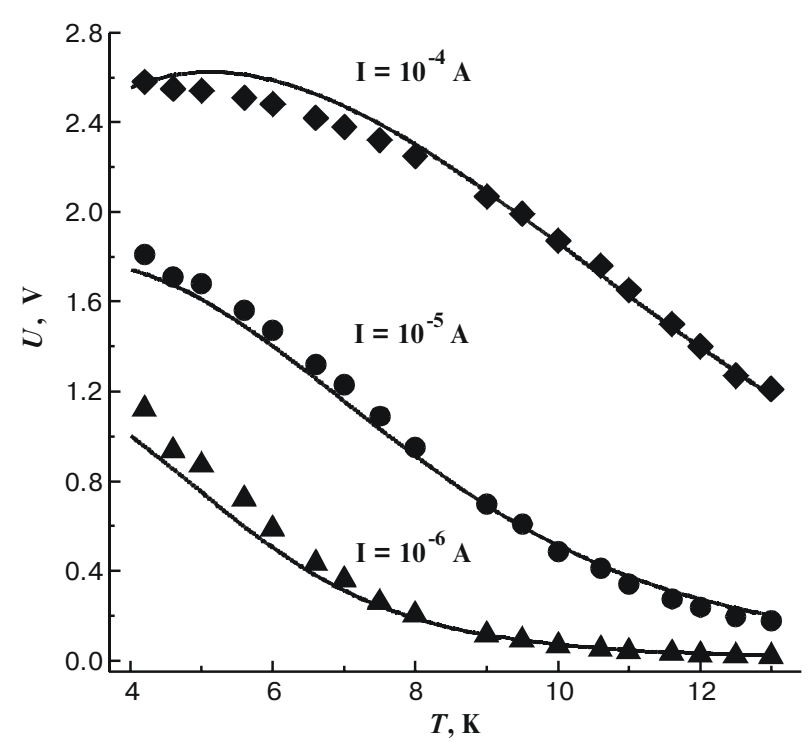

Fig. 4. Experimental and theoretical (expression (12)) thermometric characteristic of resistor with $L=17.5 \mu \mathrm{m}$ at three operating current $I$ values.
The above expression enables one to analyze the effect of hopping conductivity on the resistor thermometric characteristic. Using expression (10), one can write down the relation between $E$ and $T$ at $I=$ const:

$$
E \exp \left(\alpha_{0} T^{-5 / 4} E\right)=\left(\frac{I}{S \sigma_{00}}\right)\left(\frac{T_{0}}{T}\right)^{\frac{2-v}{4}} \exp \left(\left(\frac{T_{0}}{T}\right)^{\frac{1}{4}}\right)
$$

The solution to the equation (11) is expressed through the Lambert function $L[W(T)]$ :

$E(T)=\frac{T^{5 / 4}}{\alpha_{0}} L[W(T)]$,

where $W(T)=\frac{I}{S \sigma_{00}} \frac{\alpha_{0}}{T^{5 / 4}}\left(\frac{T_{0}}{T}\right)^{\frac{v-2}{4}} \exp \left(\left(\frac{T_{0}}{T}\right)^{\frac{1}{4}}\right)$.

Shown in Fig. 4 are both the experimental thermometric characteristics and calculated (from (12)) $U(T)$ curves. One can see that the model used enables one to describe the experimental thermometric characteristics over a wide $\left(10^{-6}\right.$ to $\left.10^{-4} \mathrm{~A}\right)$ operating current range. The temperature dependencies of resistor responsivity, $d U / d T$, calculated from expression (12) are given in Fig. 5. Their feature is presence of a peak; this is also observed in experiment. From expression (10) it follows that responsivity due to hopping conductivity is negative over the temperature range studied. Its magnitude is determined by the hopping mechanism parameters $\alpha_{0}, T_{0}$ and $v$.



Fig. 5. Theoretical temperature dependence of responsivity for test resistor with $L=17.5 \mu \mathrm{m}$ at three operating current $I$ values (expression (12)). The experimental positions of responsivity peaks are marked with asterisks. 
Thus, allowance for non-ohmic character of the Mott conductivity enables one to adequately describe the experimentally observed resistor thermometric characteristics.

\section{Effect of model parameters on the resistor thermometric characteristics}

Using expressions (7) and (10), one can present the resistor $I-V$ curves as

$$
I=(S / L) \sigma_{0} U \exp \left[-\left(T_{0} / T\right)^{1 / 4}+\alpha_{1} T^{-5 / 4} U\right]
$$

Let us consider the effect of the Mott conductivity non-ohmic character on the resistor thermometric characteristic and perform an analysis of the factors resulting in peak appearance on the temperature dependence of responsivity.

1. The non-ohmic character of the $I-V$ curves for the samples studied in the Mott hopping conductivity region is determined by the parameter $\alpha_{1}(T)=C e(a / 2 L) T_{0}^{1 / 4} k^{-1}$, as well as a ratio between the two terms in the exponent (13). This ratio depends on the impurity band parameters $T_{0}$ and $a$ (they determine both the charge carrier energy $\varepsilon_{0}$ and hopping range $R$ ), as well as the current (electric field) and temperature. At low currents the effect of the Mott conductivity non-ohmic character decreases. To illustrate the impact of this effect on formation of the thermometric characteristic, let us consider two resistors (whose hopping conductivity parameters are the same) differing in the spacing between contacts. In this case, at the same voltage drop, the electric field $E$ (as well as current $I$ ) in the longer resistor will be less than the same quantities in another one. The theoretical and experimental thermometric characteristics for two resistors of different lengths that are presented in Fig. 6a demonstrate the effect of non-ohmic conductivity on the form of thermometric characteristics. One can see from Fig. $6 \mathrm{a}$ (curve 1) that an increase in electric field leads to a more smooth temperature dependence of responsivity. Therefore one can affect both the thermometric characterisctic form and responsivity value (through a change in nonohmic character of Mott conductivity) by varying the intercontact spacing and, correspondingly, current (or electric field) value. It should be also noted that an increase in the spacing between the resistor contacts (at a fixed current value) results in a shift of thermometric characteristic along the $U$-axis.

2. An analysis of the theoretical thermometric characteristic shows that a peak on the temperature dependence of responsivity (see Fig. 5) stems from the non-ohmic character of Mott conductivity in the resistors studied. Both the height and position of the peak depends on the parameter $T_{0}$ (determined by the density of states at the Fermi level that is related to the impurity concentration) and operating current value. At low current (electric field) values the non-ohmic character of conductivity decreases in importance, and the peak disappears. The dependence of temperature value that corresponds to the highest responsivity on the density of states at the Fermi level is shown in Fig. 7. It is of interest that an increase in the index $v$ (that determines correlation radius for percolation cluster) up to 0.99 [6] results in disappearance of the
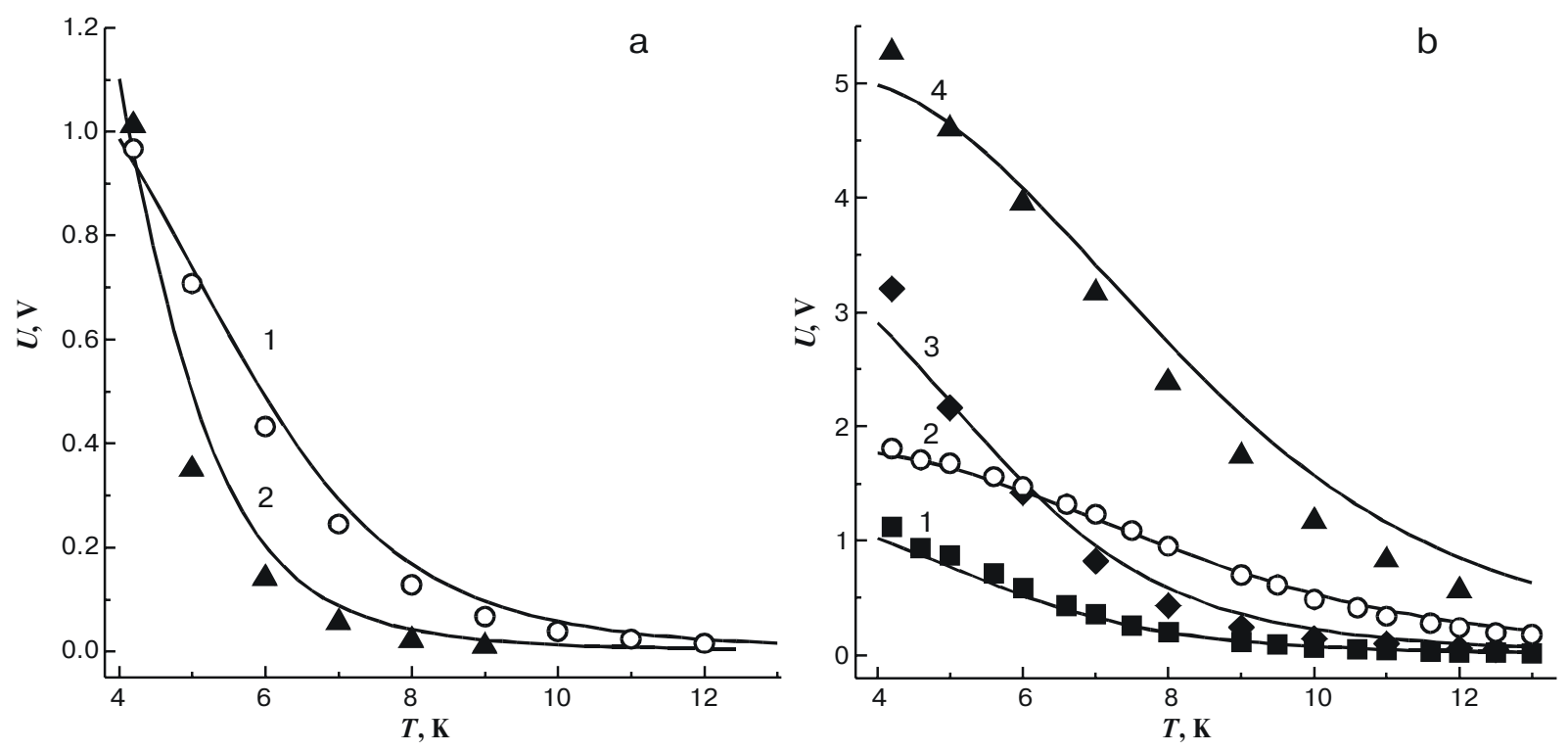

Fig. 6 a). Experimental and theoretical thermometric characteristics for two resistors (differing in length $L$ ) at the same voltage drop at a temperature of $4.2 \mathrm{~K}$ : dots - experiment, lines - theory. $1-L=17.5 \mu \mathrm{m}, I=1.8 \cdot 10^{-6} \mathrm{~A} ; 2-L=52.5 \mu \mathrm{m}, I=8.5 \cdot 10^{-8} \mathrm{~A}$.

b). Experimental and theoretical thermometric characteristics for two resistors (differing in length $L$ ) at two operating current values: dots - experiment, lines - theory. $L=17.5 \mu \mathrm{m}: 1-I=10^{-6} \mathrm{~A} ; 2-I=10^{-5} \mathrm{~A} ; L=52.5 \mu \mathrm{m}: 3-I=10^{-6} \mathrm{~A} ; 4-I=10^{-5} \mathrm{~A}$. 
Yu. M. Shwarts et al.: Non-ohmic Mott conductivity and thermometric characteristics ...

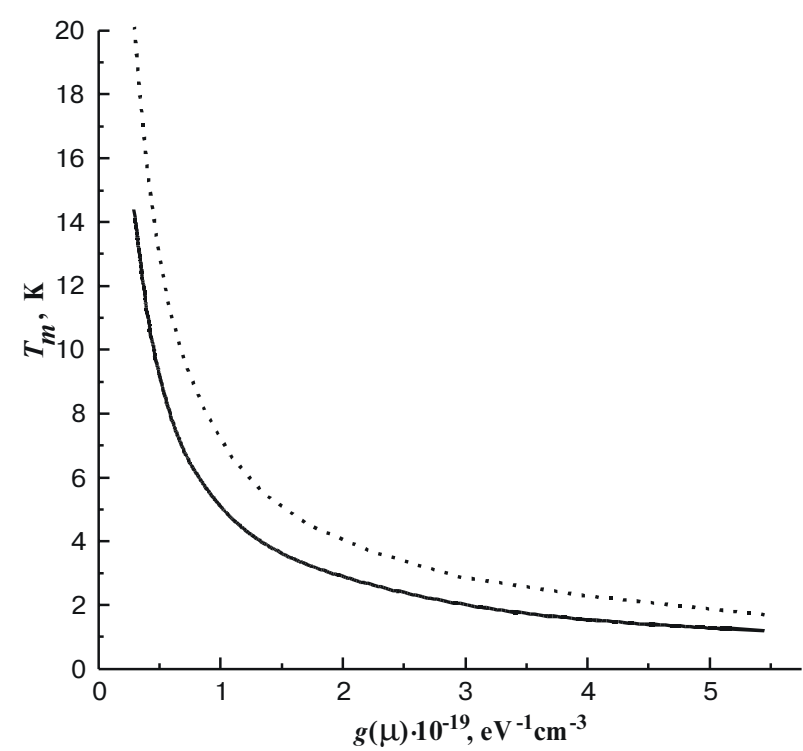

Fig. 7. Temperature at which responsivity peaks as a function of the density of states at the Fermi level for two operating current $I$ values: $10^{-6} \mathrm{~A}$ (full curve) and $10^{-5} \mathrm{~A}$ (dotted curve).

peak. Thus, the temperature dependence of the pre-exponential factor in the expression for hopping conductivity also is of importance in formation of the responsivity nonmonotonic behavior at low temperatures.

\section{Conclusions}

The presented results of our investigations of heavily doped diffused silicon structures prove that at liquid helium temperatures and low electric fields their conductivity is of hopping type. Its temperature dependence is $\sigma \sim \exp \left(T^{1 / 4}\right)$. The feature of current flow in the structures studied is that Mott conductivity is non-ohmic at electric fields $30 \cdot(T)^{5 / 4} \mathrm{~V} / \mathrm{cm}<E<500 \cdot T \mathrm{~V} / \mathrm{cm}$. This is due to an electron energy increase at the hopping range.

The model for conductivity chosen by us made it possible to explain the features of observed thermometric characteristics, such as (i) non-monotonic character of responsivity in the 4.2 to $13 \mathrm{~K}$ temperature range; (ii) presence of a peak on the $d U / d T$ curve (whose height may reach $200 \mathrm{mV} / \mathrm{K}$ ); (iii) effect of operating current value and resistor length on the responsivity and form of thermometric characteristic. It is shown that position of the responsivity peak essentially depends on the density of states at the Fermi level.

The results obtained should be taken into account when developing wide-range cryogenic diode temperature sensors.

\section{References}

1. L.G. Rubin, Cryogenic thermometry: a review of progress since 1982 // Cryogenics 37(7), pp. 341-356 (1997).

2. Yu.M. Shwarts, V.L. Borblik, N.R. Kulish, V.N. Sokolov, M.M. Shwarts, E.F. Venger, Silicon diode temperature sensor without a kink of the response curve in cryogenic temperature region // Sensors and Actuators A, Physical 76(1-3), pp. 107111 (1999).

3. I. Shlimak, M. Kaveh, R. Ussyshkin et al., Temperature-induced smearing of the Coulomb gap: experiment and computer simulation // Phys. Rev. Lett. 75 (26), pp.4764-4767 (1995).

4. N.F. Mott, E.A. Davies, Electronic Processes in Non-Crystalline Materials, $2^{\text {nd }}$ Ed., Clarendon Press, Oxford (1979).

5. X. Liu, A.Sidorenko, S. Wagner, P. Ziegler, H.V. Lohneysen, Electronic transport processes in heavily doped uncompensated and compensated silicon as probed by the thermoelectric power // Phys. Rev. Lett. 77 (16), pp.3395-3398 (1996).

6. B.I. Shklovskii, A.L. Efros, Electronic Properties of Doped Semiconductors, Springer Verlag, Berlin (1984).

7. M. Pollak, I. J. Riess, Hopping conduction in amorphous semiconductors // Phys. C 9(12), pp. 2325-2339 (1976).

8. R.M. Hill, Hopping conductivity in amorphous solids // Phil. Mag. 24(192), pp. 1307-1325 (1971).

9. A.G. Zabrodskii, A.I. Ionov, I.S. Shlimak, Effect of electric and magnetic fields on charge transfer in heavily doped and compensated semiconductors (in Russian) // Fiz. Tekhn. Poluprov. 8(3), pp. 503-508 (1974).

10. I.N. Timchenko, V.A. Kasiyan, D.D. Nedeoglo, A.A Simashevich, Variable-range hopping conductivity in $n-\mathrm{ZnSe}$ crystals in moderate electric fields (in Russian) // Fiz. Tekhn. Poluprov. 23(2), pp. 240-243 (1989).

11. E.I. Levin, B.I. Shklovskii, Low-temperature hopping conductivity in high electric fields (in Russian) // Fiz. Tekhn. Poluprov. 18(5), pp. 856-864 (1984). 